\title{
Gravitational wave signals of dark matter freeze-out
}

\author{
Danny Marfatia ${ }^{a}$ and Po-Yan Tseng ${ }^{b}$ \\ ${ }^{a}$ Department of Physics and Astronomy, University of Hawaii, \\ 2505 Correa Rd., Honolulu, HI 96822, U.S.A. \\ ${ }^{b}$ Department of Physics and IPAP, Yonsei University, \\ 50 Yonsei-ro, Seoul 03722, Republic of Korea \\ E-mail: dmarf8@hawaii.edu, tpoyan1209@gmail.com
}

ABSTRACT: We study the stochastic background of gravitational waves which accompany the sudden freeze-out of dark matter triggered by a cosmological first order phase transition that endows dark matter with mass. We consider models that produce the measured dark matter relic abundance via (1) bubble filtering, and (2) inflation and reheating, and show that gravitational waves from these mechanisms are detectable at future interferometers.

KeYwords: Beyond Standard Model, Cosmology of Theories beyond the SM

ARXIV EPRINT: 2006.07313 


\section{Contents}

1 Introduction 1

2 Bubble filtering 3

3 Bubble wall velocity 4

$\begin{array}{lll}4 & \text { Gravitational wave production } & 6\end{array}$

5 Models $\quad 6$

$\begin{array}{lll}5.1 & \text { Scalar quartic model } & 7\end{array}$

$5.2 \mathrm{SU}(2)_{X}$ model 9

5.2.1 Dark matter abundance 11

$\begin{array}{ll}\text { 5.2.2 Gravitational wave signals } & 12\end{array}$

6 Summary 13

\section{Introduction}

The identity of dark matter (DM) and its production mechanism are among the most important open questions in physics. In the weakly interacting massive particle (WIMP) paradigm, with its thermal freeze-out mechanism, the measured DM relic density requires a WIMP mass of $\mathcal{O}\left(10-10^{3}\right) \mathrm{GeV}$, and an electroweak-scale DM annihilation cross section. The decoupling temperature $T_{\text {dec }}$ is related to the DM mass $m_{\chi}$ by $T_{\text {dec }} \simeq m_{\chi} / 24$. The vanilla version of this scenario had been challenged by the non-observation of WIMPs in DM direct detection searches. Alternative scenarios for DM production in the early universe often assume a DM sector that is out of thermal equilibrium with the standard model (SM) sector. For example, DM may be produced in the decays of a heavy particle $[1,2]$. DM may also be produced by freeze-in through the feeble annihilation of particles which are thermalized with the SM bath [3-5]. However, in all of the above scenarios, the DM mass is constant during DM production.

The discovery of the $125 \mathrm{GeV}$ SM-like Higgs boson $h$ at the Large Hadron Collider (LHC) [6, 7] consolidates spontaneous symmetry breaking as the mechanism that gives the SM particles their mass. The Higgs mechanism gives the simple relation $m_{f}=y_{f} \cdot v_{\mathrm{SM}}$ between the fermion mass $m_{f}$ and its Yukawa coupling to the Higgs boson $y_{f}$, where $\langle h\rangle \equiv$ $v_{\mathrm{SM}} \simeq 246 \mathrm{GeV}$ is the vacuum expectation value (VEV) of the SM Higgs. A picture of the universe going through an electroweak phase transition because finite temperature effects modify its scalar potential as the universe cools down, emerges. Before the phase transition, when all the SM particles are massless, the global minimum of the scalar potential is located 
at $\langle h\rangle=0$. After the phase transition, the global minima of the potential shift to nontrivial values $\langle h\rangle \neq 0$, which gives mass to the SM particles. In the SM, the electroweak phase transition is found non-perturbatively to be a smooth crossover [8,9]. However, since we do not fully understand the entire structure of the scalar potential of the $125 \mathrm{GeV}$ Higgs boson, and since the existence of additional scalars is a possibility, the nature of the transition is unknown.

The DM mass may be generated by a similar mechanism [10-14]. The mass originates from its couplings to a scalar, which obtains a non-trivial VEV in the early universe, so that massless DM becomes massive during the phase transition. The scalar may or may not be the $125 \mathrm{GeV}$ Higgs boson. We consider a first order phase transition (FOPT) in the early universe, with vacuum bubbles nucleated at temperature $T_{\star}$, which ends with the expanding bubbles populating the entire universe; until we discuss inflationary supercooling, we do not differentiate between the nucleation temperature $T_{n}$ and the temperature $T_{\star}$ at which gravitational waves are produced. The symmetric and broken phases are located outside and inside the bubbles, respectively. The massless DM particles outside the bubbles become massive when they enter the bubbles. Only massless DM particles that carry kinetic energy larger than $m_{\chi}$ can penetrate the bubble walls and become massive. DM inside the bubbles abruptly decouples from the thermal bath if $T_{\star}<T_{\mathrm{dec}}$. The result is that the bubbles filter out a certain amount of DM and determine the DM relic abundance [13, 14]. The massless DM outside the bubbles remains thermalized with SM radiation. It is also possible that all the massless DM particles enter the bubbles after being diluted by a period of inflation, which determines the relic abundance [11]. DM particles with insufficient kinetic energy to enter the bubbles, bounce back to the symmetric phase and slow down the bubble expansion by applying pressure on the bubble walls.

The value of $m_{\chi} / T_{\star}$ needed to produce the correct DM relic abundance depends on the velocity of the bubble walls $v_{w}$. For instance, $T_{\star} \simeq m_{\chi} / 30$ for $m_{\chi}=1 \mathrm{TeV}$ and $v_{w}=0.01$, which satisfies $T_{\star}<T_{\mathrm{dec}}$. Note that DM freeze-out induced by a FOPT can easily accommodate DM masses above a $\mathrm{PeV}$, which is beyond the current sensitivities of DM direct detection and LHC searches.

In this paper, we focus on gravitational wave (GW) signals of sudden DM freeze-out caused by a FOPT during which DM mass is generated. Because the power and frequency spectrum of the GW signal is model dependent, we choose two example models, i) Scalar Quartic Model [15-18] and ii) $\mathrm{SU}(2)_{X}$ Model $[10,11]$, to demonstrate that in parameter space regions that yield the observed DM relic abundance, a detection is possible at future GW interferometers. In the Scalar Quartic Model, the DM abundance is determined by bubble filtering, while in the $\mathrm{SU}(2)_{X}$ Model, the DM abundance is set by inflation and reheating.

The paper is organized as follows. Bubble filtering is described in section 2, and computations of the bubble wall velocity are detailed in section 3. In section 4, we list the contributions to GW spectra from various processes. We calculate the GW signals for the two example models in section 5 , and summarize in section 6 . 


\section{Bubble filtering}

During the FOPT and bubble expansion, massless (massive) DM particles are located outside (inside) the bubble, and momentum conservation must be satisfied at the bubble wall. An incident DM particle enters the bubble if it carries kinetic energy larger than its mass inside the bubble. Otherwise, the massless DM particle is reflected and stays outside the bubble. If a thermal flux of $\chi$ is incident on the wall, the number density of DM particles that enter the bubble is [14]

$$
n_{\chi}^{\text {in }}=n_{\bar{\chi}}^{\text {in }} \simeq \frac{g_{\mathrm{DM}} T_{\star}^{3}}{\gamma_{w} v_{w}}\left(\frac{\gamma_{w}\left(1-v_{w}\right) m_{\chi} / T_{\star}+1}{4 \pi^{2} \gamma_{\omega}^{3}\left(1-v_{w}\right)^{2}}\right) e^{-\frac{\gamma_{w}\left(1-v_{w}\right) m_{\chi}}{T_{\star}}} .
$$

where $\gamma_{w}$ is the Lorentz boost factor of the wall in the rest frame of the plasma, $g_{\mathrm{DM}}$ is the number of spin states of the DM particle, and the DM distribution has been approximated to be Boltzmann. In the non-relativistic limit, $v_{w} \rightarrow 0$, filtering strongly suppresses the DM number density inside the bubble as $e^{-m_{\chi} / T_{\star}}$. In the relativistic limit, $m_{\chi} /\left(\gamma_{w} T_{\star}\right) \rightarrow 0$, the number density $\sim e^{-m_{\chi} /\left(2 \gamma_{w} T_{\star}\right)}$, so there is very little filtering and $n_{\chi}^{\text {in }}$ approaches the equilibrium number density outside the bubble, $\left.n_{\chi}^{\mathrm{eq}}\right|_{T=T_{\star}}=g_{\mathrm{DM}} T_{\star}^{3} / \pi^{2}$.

If $T_{\star}$ is lower than the thermal decoupling temperature $T_{\mathrm{dec}}$, the DM inside the bubble is already decoupled from the thermal bath and makes up the DM relic abundance, On the other hand, if $T_{\star}>T_{\mathrm{dec}}$, the DM filtered by the bubble wall remains in thermal equilibrium and the relic abundance is determined by standard thermal freeze-out with $m_{\chi} / T_{\text {dec }} \simeq 24{ }^{1}$

The DM abundance today can be calculated by dividing $n_{\chi}^{\text {in }}+n_{\bar{\chi}}^{\text {in }}\left(\right.$ at $\left.T_{\star}\right)$ by the entropy density $s=\left(2 \pi^{2} / 45\right) g_{\star S} T^{3}$, where $g_{\star S}$ is the effective number of relativistic degrees of freedom associated with entropy, and normalizing to the critical density, $\rho_{c}=3 H_{0}^{2} M_{\mathrm{pl}}^{2}[13]$ :

$$
\Omega_{\mathrm{DM}} h^{2} \simeq 6.29 \times 10^{8} \frac{m_{\chi}\left(n_{\chi}^{\mathrm{in}}+n_{\bar{\chi}}^{\mathrm{in}}\right)}{\mathrm{GeV}} \frac{1}{g_{\star S} T_{\star}^{3}} .
$$

Using eq. (2.1), this can be simplified to

$$
\Omega_{\mathrm{DM}} h^{2} \simeq \begin{cases}1.27 \times 10^{8}\left(\frac{m_{\chi}}{\mathrm{GeV}}\right)\left(\frac{g_{\mathrm{DM}}}{g_{\star S}}\right)\left(\frac{m_{\chi}}{2 \gamma_{w} T_{\star}}+1\right) e^{-\frac{m_{\chi}}{2 \gamma_{w} T_{\star}},} & \text { for } v_{w} \rightarrow 1 \\ 3.19 \times 10^{7}\left(\frac{m_{\chi}}{\mathrm{GeV}}\right)\left(\frac{g_{\mathrm{DM}}}{g_{\star S}}\right)\left(\frac{1}{v_{w}}\right)\left(\frac{m_{\chi}}{T_{\star}}+1\right) e^{-\frac{m_{\chi}}{T_{\star}}}, & \text { for } v_{w} \rightarrow 0 .\end{cases}
$$

Then, $\Omega_{\mathrm{DM}} h^{2} \simeq 0.11$ requires

$$
\begin{aligned}
\frac{m_{\chi}}{2 \gamma_{w} T_{\star}}-\ln \left(\frac{m_{\chi}}{2 \gamma_{w} T_{\star}}\right)-\ln \left(g_{\mathrm{DM}}\right)-\ln \left(\frac{m_{\chi}}{\mathrm{GeV}}\right) & \simeq 16.2, \text { for } v_{w} \rightarrow 1 \\
\frac{m_{\chi}}{T_{\star}}-\ln \left(\frac{m_{\chi}}{T_{\star}}\right)+\ln \left(v_{w}\right) & \simeq 22, \quad \text { for } v_{w} \rightarrow 0 .
\end{aligned}
$$

\footnotetext{
${ }^{1}$ Note that even with the FOPT, $T_{\text {dec }}$ is obtained by equating the Hubble expansion rate $H$ and the thermal averaged DM annihilation rate, $\Gamma=\langle\sigma v\rangle n^{\text {in,eq }}[13]$. We assume that the SM makes a dominant contribution to the light degrees of freedom so that $m_{\chi} / T_{\mathrm{dec}} \simeq 24$ with logarithmic corrections that depend on $m_{\chi}, T_{\star}$ and the DM coupling.
} 

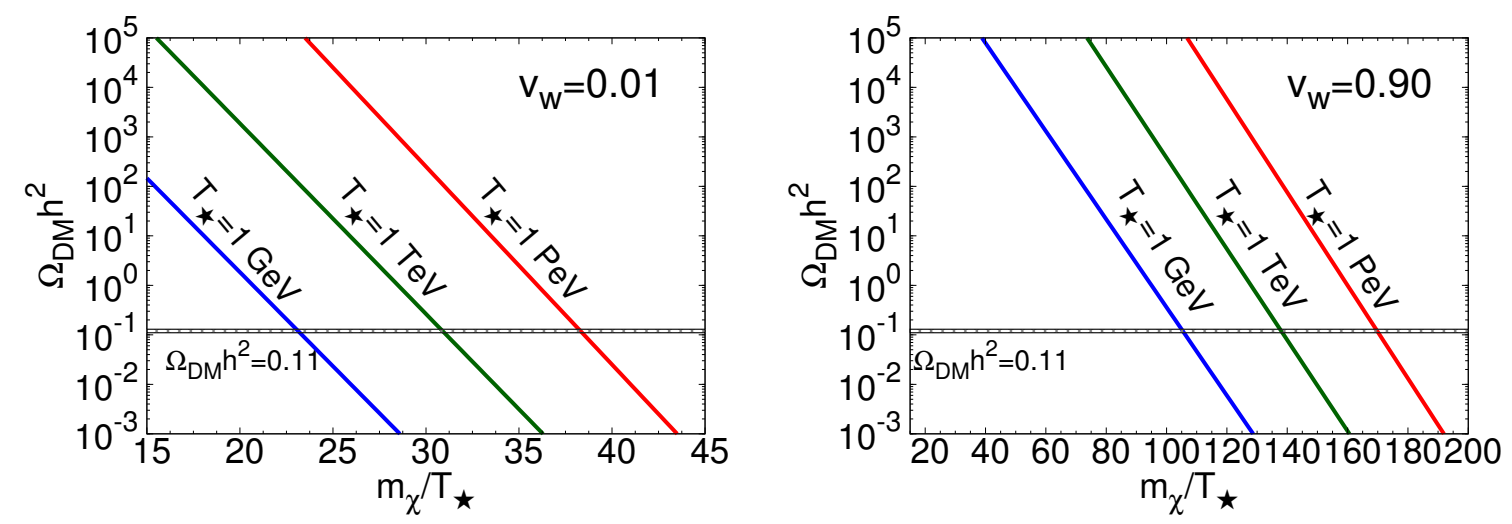

Figure 1. The DM relic abundance after bubble filtering for non-relativistic and relativistic bubble wall velocities.

For example, for $v_{w} \rightarrow 1$, taking $m_{\chi} \approx 1 \mathrm{TeV}$ and $g_{\mathrm{DM}}=2$, requires

$$
\frac{m_{\chi}}{2 \gamma_{w} T_{\star}} \simeq 27
$$

to give the measured DM relic abundance, $\Omega_{\mathrm{DM}} h^{2} \simeq 0.11$.

The DM relic abundances for three values of $T_{\star}$ and relativistic and non-relativistic wall velocities are shown in figure 1 . The left-panel shows that for small $v_{w}, \Omega_{\mathrm{DM}} h^{2} \simeq 0.11$ if $20 \lesssim m_{\chi} / T_{\star} \lesssim 40$ and $1 \mathrm{GeV} \lesssim T_{\star} \lesssim 1 \mathrm{PeV}$. For $v_{w} \rightarrow 1$ (right panel), because bubble filtration is not efficient, larger values, $100 \lesssim m_{\chi} / T_{\star} \lesssim 170$, in the exponent of eq. (2.1) are needed to suppress the DM number density. That larger $T_{\star}$ requires larger $m_{\chi} / T_{\star}$ can be understood by combining eq. (2.1) and (2.2): $\Omega_{\mathrm{DM}} h^{2} \propto T_{\star}\left(m_{\chi} / T_{\star}\right)^{2} e^{-m_{\chi} / T_{\star}}$.

\section{Bubble wall velocity}

We consider a fermionic or bosonic DM particle $\chi$ that couples to a scalar $\eta$ (that could be the SM Higgs or a new particle) with coupling $g_{\chi}$ (not to be confused with $g_{\mathrm{DM}}$, the number of spin states). The scalar undergoes a FOPT at temperature $T_{\star}$, during which the VEV jumps from $\langle\eta\rangle=0$ to $\langle\eta\rangle=v_{\eta}$. Nucleation starts at $T_{\star}$, and the bubbles expand and merge until the entire universe is populated with the $v_{\eta}$ phase. During the bubble expansion two phases coexist. Inside the bubbles $\langle\eta\rangle=v_{\eta}$, and DM gets a mass $m_{\chi} \simeq g_{\chi} v_{\eta}$. Outside the bubbles, $\chi$ is massless because $\langle\eta\rangle=0$. Bubble filtering occurs as described in the previous section.

DM particles that are reflected by the bubble wall exert pressure $P$ on it, and slow down the bubble wall velocity, which is given by the equilibrium condition $\Delta V=P$, where $\Delta V$ is the potential energy difference between the false and true vacua. The strength of the phase transition is defined in terms of the latent heat of the transition,

$$
\alpha \equiv \frac{\left.\left(1-T \frac{\partial}{\partial T}\right) \Delta V\right|_{T=T_{\star}}}{\rho_{\mathrm{rad}}\left(T_{\star}\right)},
$$

where the radiation energy density, $\rho_{\mathrm{rad}}(T)=\pi^{2} g_{\star} T^{4} / 30$, with $g_{\star}$ the number of effectively massless degrees of freedom at temperature $T$. For the SM, far above the electroweak scale, 
$g_{\star} \simeq 106.75$. Note that the derivative term in eq. (3.1) is negligible for strong, supercooled transitions, as is the case for the $\mathrm{SU}(2)_{X}$ Model.

In the ultrarelativistic limit, the pressure on the bubble wall can be obtained from the difference in the number of light degrees of freedom inside and outside the bubble $[14,19,20]$ :

$$
P=\frac{d_{n} g_{\star} \pi^{2}}{90}\left(1+v_{w}\right)^{3} \gamma_{\omega}^{2} T_{\star}^{4},
$$

where the ratio of the number of light degrees of freedom is

$$
d_{n} \equiv \frac{1}{g_{\star}}\left[\sum_{0.2 M_{i}>\gamma_{w} T_{\star}}\left(g_{i}^{b}+\frac{7}{8} g_{i}^{f}\right)\right],
$$

with $g_{i}^{b}$ and $g_{i}^{f}$, the number of degrees of freedom of the bosons and fermions, respectively. If particle $i$ of the thermal plasma gains mass $M_{i}$ inside the bubble and $0.2 M_{i} \gtrsim \gamma_{w} T_{\star}$, then most of the $i$ particles fail to penetrate the wall and instead exert pressure on it [14]. If $i$ is fermionic DM with $g_{\mathrm{DM}}=2$, then $d_{n} \simeq 0.032$ including particle and antiparticle contributions. Therefore, once $\alpha$ is known from the scalar potential, $v_{w}$ can be obtained by solving the equation, $\Delta V=P$ :

$$
\alpha=\frac{d_{n}}{3}\left(1+v_{w}\right)^{3} \gamma_{\omega}^{2}
$$

In the limit $v_{w} \rightarrow 1$, with $d_{n}=0.032$, we find $\alpha \simeq 0.085 \gamma_{\omega}^{2}$ from eq. (3.2). Eliminating $\gamma_{w}$ from eq. (2.5) yields the condition,

$$
\frac{m_{\chi}}{\sqrt{\alpha} T_{\star}} \simeq \frac{g_{\chi} v_{\eta}}{\sqrt{\alpha} T_{\star}} \simeq 185
$$

to produce the measured relic abundance for $m_{\chi} \approx 1 \mathrm{TeV}$. If we assume $g_{\chi} \simeq \mathcal{O}(1)$, a large $v_{\eta} / T_{\star} \gtrsim \mathcal{O}(10)$ and small $\alpha \lesssim \mathcal{O}(0.1)$ is required.

A precise computation of the bubble wall velocity outside the ultrarelativistic regime is beyond the scope of this paper. For bubble wall velocities faster than the speed of sound in the plasma $(1 / \sqrt{3})$, but not ultrarelativistic, we use the approximation [21],

$$
v_{w}=\frac{\frac{1}{\sqrt{3}}+\sqrt{\alpha^{2}+\frac{2}{3} \alpha}}{1+\alpha} .
$$

For $v_{w} \rightarrow 1 / \sqrt{3}$ (i.e., $\alpha \rightarrow 0$ ), the condition for $\Omega_{\mathrm{DM}} h^{2}=0.11$ is

$$
\frac{m_{\chi}}{T_{\star}}=\frac{g_{\chi} v_{\eta}}{T_{\star}} \simeq 75
$$

according to eqs. (2.1) and (2.2).

The wall velocity in eq. (3.4) is fixed by the Chapman-Jouguet condition for fluid expansion in chemical combustion. Since this condition is generally not fulfilled [19, 22], we consider a range of velocities around the value of eq. (3.4) to parameterize the uncertainty in the predicted GW signal. 


\section{Gravitational wave production}

A FOPT generates GWs from three processes [23]: i) Bubble collisions. ii) Sound waves in the plasma following bubble collisions and before the kinetic energy is dissipated by bubble expansion. iii) Magnetohydrodynamic (MHD) turbulence in the plasma after the bubble collisions. The parameters that control the signal are $v_{w}, T_{\star}$, the phase transition strength $\alpha$, the inverse of the duration of the phase transition $\beta / H_{\star}$ in units of the Hubble parameter at $T_{\star}$, all of which are model and scalar potential specific.

Our calculations of the GW spectra follow the semi-analytic treatment in refs. [19, 23, 24]. Here, we simply point out some aspects of the three contributions without regurgitating the equations used. Increasing the values of $T_{\star}$ and $\beta / H_{\star}$ increases the peak GW frequency, but the latter also suppresses the power of the GW signal. The power also decreases as $v_{w}$ is decreased. These properties are shared by all three GW contributions.

The GW contribution from bubble collisions can be calculated directly from the scalar field $\eta$ in the envelope approximation. In this approximation, an important quantity is the fraction of latent heat transformed into scalar field gradient energy, $\kappa_{\eta}$.

GWs are produced by the sound waves created during percolation. For values of $v_{w}$ not too close to the sound speed or speed of light, parametric fits to the numerically obtained GW spectrum can be found in ref. [23]. These fits include an efficiency parameter $\kappa_{v}$ for the fraction of latent heat transformed into bulk motion of the fluid, that depends on the expansion mode of the bubble. The peak frequency of the contribution from sound waves is inversely propositional to $v_{w}$.

The contribution from MHD turbulence arises when percolation transfers a $\kappa_{\text {turb }}$ fraction of the latent heat into turbulence in the plasma. This parameter is related to $\kappa_{v}$ via $\kappa_{\text {turb }} \simeq \epsilon \kappa_{v}$, where $\epsilon$ represents the fraction of bulk motion that is turbulent. The value of $\epsilon$ is still under investigation, and we conservatively take $\epsilon=0.05$ [23], which makes the contribution from MHD turbulence small.

Even in the case of significant supercooling, as shown in ref. [25], bubble walls do not runaway because of friction provided by transition radiation. In our study of the $\mathrm{SU}(2)_{X}$ Model, we explicitly check that the vacuum contribution driving the expansion dominates the pressure difference due to transition radiation across the wall. Then, the walls carry most of the energy and the GW signal arises from bubble collisions.

\section{Models}

We now investigate two example models to demonstrate that abrupt DM freeze-out produces a detectable stochastic GW background.

We consider the Scalar Quartic Model and $\mathrm{SU}(2)_{X}$ Model. Both models have a quartic term as the highest order term in their scalar potentials. However, in the former model, the effective scalar potential is composed of only one scalar field $\eta$, and may be viewed as approximating a multi-field potential. There may be thermal or non-thermal contributions to the cubic term from new particles that are not heavy enough to be integrated out [16]. In this model, the DM candidate is unspecified. On the other hand, the $\mathrm{SU}(2)_{X}$ Model has the 
SM gauge group with an extra $\mathrm{SU}(2)_{X}$, and the scalar potential at the Planck scale is assumed to only permit quartic terms built from the SM scalar doublet $H$ and a scalar doublet $S$ under $\mathrm{SU}(2)_{X}$. The absence of quadratic terms renders the model dimensionless at tree level. The quadratic terms and electroweak scale are dynamically generated [26, 27], and the $\mathrm{SU}(2)_{X}$ vector bosons are automatically stable and are the DM candidates [28, 29]. A generalization of this model that includes mass terms at tree level has been studied in ref. [30].

\subsection{Scalar quartic model}

The effective scalar potential at finite temperature is $[15-18]$

$$
V_{\text {eff }}(\eta, T)=\frac{\mu^{2}+D T^{2}}{2} \eta^{2}-\xi T \eta^{3}+\frac{\lambda}{4} \eta^{4},
$$

where we have neglected non-thermal contributions to the cubic term. Many particle physics models such as the inert singlet, inert doublet, and minimal supersymmetry models, can be parametrized by the above finite-temperature effective potential. We identify $\eta$ with the SM Higgs and set the zero-temperature VEV to the SM value $v_{\eta}=v_{\mathrm{SM}}=246 \mathrm{GeV}$. Since $\xi$ is not the Higgs trilinear coupling, but the $T$-independent coefficient of the $\eta^{3}$ term of the high- $T$ expansion of the Higgs effective potential, we take $\xi$ as a free parameter. Then the critical temperature is [16]

$$
T_{c}=\frac{2}{\lambda D-2 \xi^{2}}\left[\frac{\sqrt{\left.\lambda D\left(\lambda D-2 \xi^{2}\right) T_{o}^{2}\right)}}{2}\right],
$$

where

$$
T_{0}^{2}=-\frac{\mu}{D}=\frac{\lambda}{D} v_{\mathrm{SM}}^{2},
$$

is the temperature when the potential barrier vanishes. The two minima are

$$
\langle\eta\rangle=0, \quad \frac{3 \xi T}{2 \lambda}\left[1+\sqrt{1-\frac{4 \lambda\left(\mu^{2}+D T^{2}\right)}{9 \xi^{2} T^{2}}}\right] \equiv v_{\eta} .
$$

There are three independent parameters $\xi, D$, and $\lambda$ in the above effective potential. For simplicity, we fix $\lambda=0.1$ in following analysis.

The nucleation temperature $T_{n}$ is determined by requiring the bounce action $S_{3}\left(T_{n}\right) / T_{n} \simeq 142$, when the vacuum tunneling rate equals the Hubble expansion rate [15]. We adopt the following analytic approximation from ref. [18]:

$$
\frac{S_{3}}{T}=\frac{64 \sqrt{2} \pi}{81} \frac{\xi}{\lambda^{3 / 2}}(2-\delta)^{-2}\left(\beta_{1} \delta+\beta_{2} \delta^{2}+\beta_{3} \delta^{3}\right),
$$

where $\delta \equiv \lambda\left(\mu^{2}+D T^{2}\right) /(\xi T)^{2}$, and $\beta_{1}=8.2983, \beta_{2}=-5.5330$ and $\beta_{3}=0.8180$ are the results of a numerical fit. The expression is valid for $0<\delta<2$ which corresponds to $T_{0}<$ $T<T_{c}$. We choose $T_{\star}=T_{n}$ to compute $\alpha$ and $\beta / H_{\star}=d\left(S_{3} / T\right) /\left.d(\ln T)\right|_{T=T_{n}}[15]$. Figure 2 shows that in only narrow parameters region (for example around $(D, \xi) \simeq(18,0.85)$ ), is $v_{\eta} / T_{\star}$ large enough $(\sim 25)$ as dictated by eq. (3.5), to obtain the measured DM relic abundance for $g_{\chi} \simeq \sqrt{4 \pi}$. 

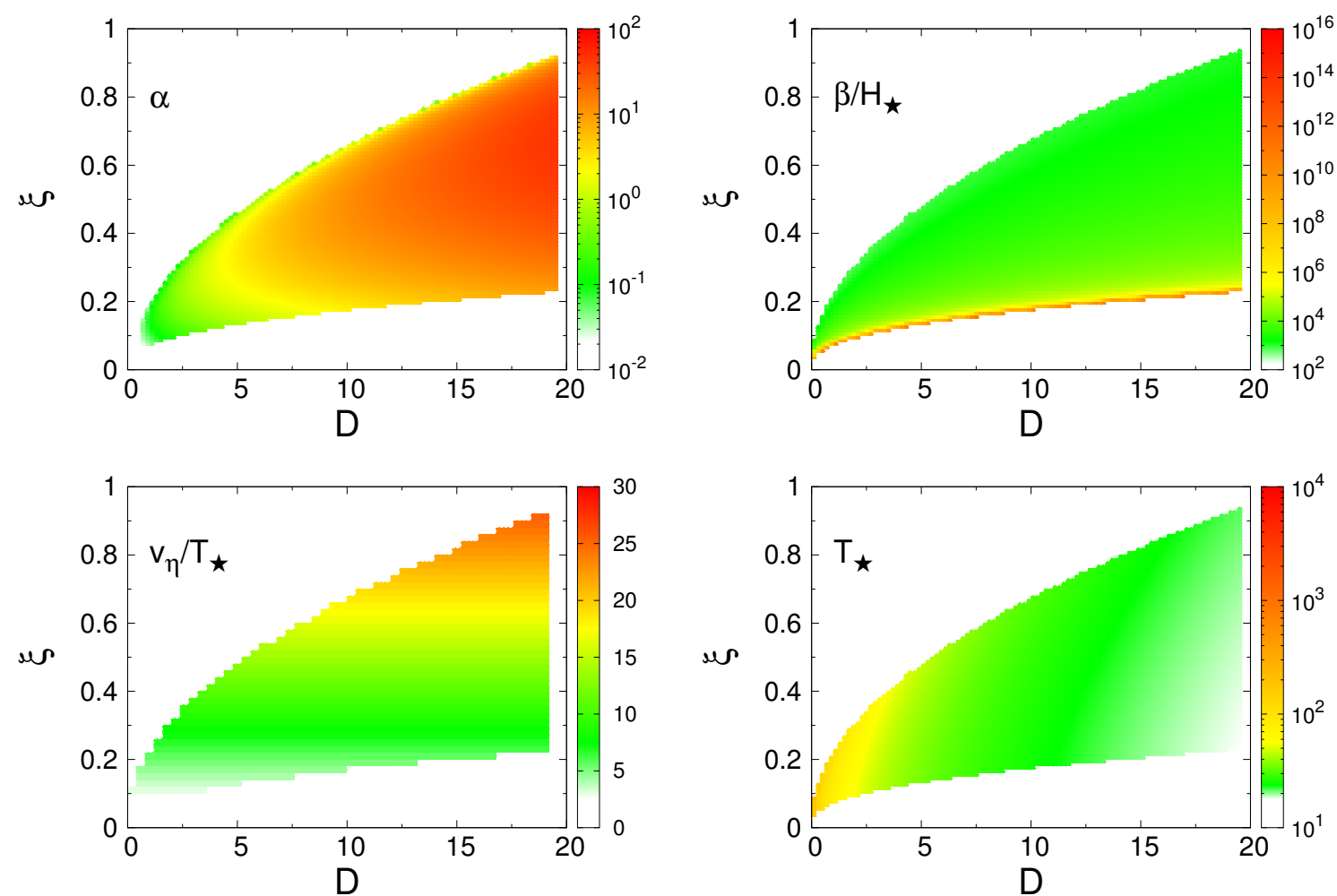

Figure 2. The parameters $\alpha, \beta / H, v_{\eta} / T_{\star}$, and $T_{\star}$ for the Scalar Quartic Model with $\lambda=0.1$.
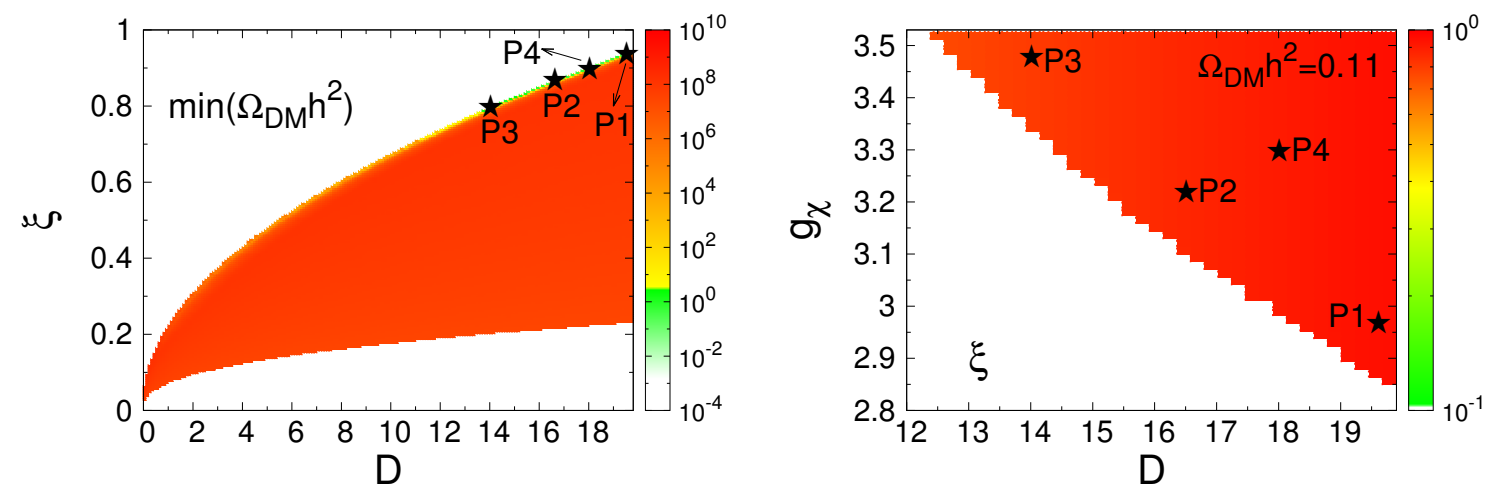

Figure 3. Scalar Quartic Model. Left: minimal values of $\Omega_{\mathrm{DM}} h^{2}$ in the $(D, \xi)$ plane for $g_{\chi} \leq \sqrt{4 \pi}$. Right: values of $\xi$ in the $\left(D, g_{\chi}\right)$ plane for $\Omega_{\mathrm{DM}} h^{2}=0.11$. The stars mark the four benchmark points in table 1 . 


\begin{tabular}{|c|cccc|}
\hline & P1 & P2 & P3 & P4 \\
\hline$\xi$ & 0.943 & 0.863 & 0.796 & 0.901 \\
$D$ & 19.7 & 16.5 & 14.0 & 18.0 \\
$g_{\chi}$ & 2.97 & 3.22 & 3.48 & 3.31 \\
\hline$\alpha$ & 0.089 & 0.082 & 0.076 & 0.121 \\
$\beta / H_{\star}$ & 1116 & 1062 & 1015 & 1085 \\
$v_{\eta} / T_{\star}$ & 25.71 & 23.41 & 21.49 & 24.51 \\
$v_{w}$ & 0.768 & 0.763 & 0.760 & 0.791 \\
$T_{\star} / \mathrm{GeV}$ & 21.5 & 23.8 & 26.1 & 22.7 \\
$m_{\chi} / \mathrm{GeV}$ & 1642 & 1799 & 1953 & 1838 \\
\hline
\end{tabular}

Table 1. Benchmark points (with $\lambda=0.1$ ) for the Scalar Quartic Model that give $\Omega_{\mathrm{DM}} h^{2}=0.11$.

The DM relic abundance is mainly determined by bubble filtering in the Scalar Quartic Model. Because of the presence of the quadratic term at tree-level, inflationary supercooling (as for the $\mathrm{SU}(2)_{X}$ Model) does not occur. In the left panel of figure 3, we show values of the relic abundance obtained by varying $D$ and $\xi$ with $g_{\chi} \leq \sqrt{4 \pi}$. In most of the parameter space DM is overproduced. However, in the narrow green region $\alpha \lesssim 0.2$; compare with the upper-left panel of figure 2. The values of $D, \xi$ and $g_{\chi}$ for which $\Omega_{\mathrm{DM}} h^{2}=0.11$ are displayed in the right panel of figure 3 . The four benchmark points marked with stars in figure 3 are listed in table 1 .

The GW spectra for the benchmark points are shown in figure 4. The frequencies peak around $\mathcal{O}\left(10^{-3}-10^{-2}\right) \mathrm{Hz}$ because $\beta / H_{\star} \simeq \mathcal{O}(1000)$ for all four points. This puts the model out of reach of LIGO and ET. LISA, BBO and DECIGO are sensitive to all four benchmark points because they have $\alpha \simeq 0.1$ which generates a large peak signal strength, $\Omega_{\mathrm{GW}} h^{2} \sim 10^{-12}$.

\section{$5.2 \mathrm{SU}(2)_{X}$ model}

In this dimensionless model, the $\mathrm{SM}$ gauge group is extended by an $\mathrm{SU}(2)_{X}$ with gauge coupling $g_{X}$, and a scalar $S$, which transforms as a doublet under $\mathrm{SU}(2)_{X}$ and is a singlet under the SM gauge group [10, 11]. The scalar potential at tree level is

$$
\begin{aligned}
V & =\lambda_{H}|H|^{4}-\lambda_{H S}|H S|^{2}+\lambda_{S}|S|^{4}, \\
\text { where } \quad S & =\frac{1}{\sqrt{2}}\left(\begin{array}{l}
0 \\
\eta
\end{array}\right), \quad H=\frac{1}{\sqrt{2}}\left(\begin{array}{l}
0 \\
h
\end{array}\right) .
\end{aligned}
$$

$\mathrm{SU}(2)_{X}$ is spontaneously broken after $\eta$ acquires a $\mathrm{VEV}\langle\eta\rangle=v_{\eta}$. We treat the three vector bosons of $\mathrm{SU}(2)_{X}$ cumulatively as a single $\mathrm{DM}$ candidate with $g_{\mathrm{DM}}=9$ and mass $m_{\chi}=g_{X} v_{\eta} / 2$.

In this model, as the universe cools down, the universe remains trapped in the false vacuum (i.e., $\langle\eta\rangle=\langle h\rangle=0$ ) during thermal inflation due to the thermal effects. Around 


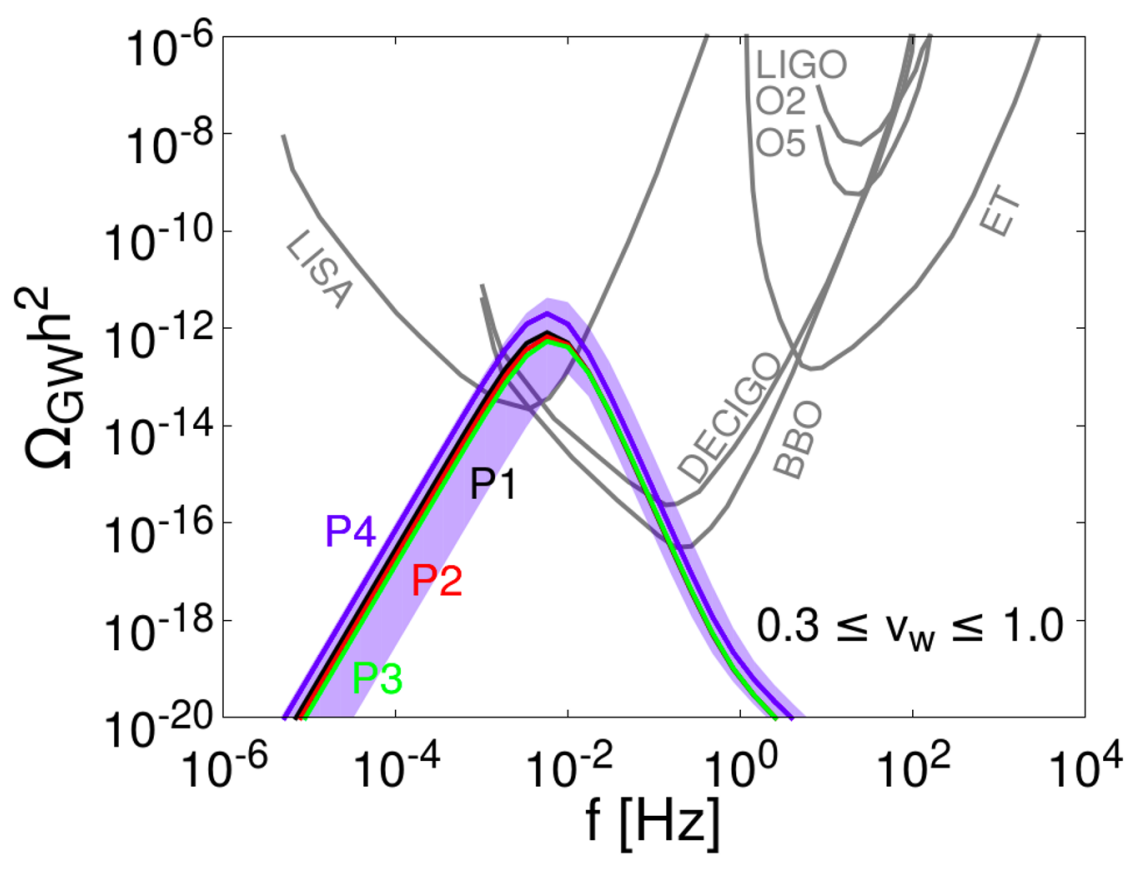

Figure 4. The GW power spectra for the benchmark points of the Scalar Quartic Model in table 1 and figure 3. The shaded band straddling the $\mathbf{P} 4$ curve shows the uncertainty in the GW spectrum by varying the bubble wall velocity in the range, $0.3 \leq v_{w} \leq 1.0$.

this vacuum, all particles are massless. When the energy of the false vacuum exceeds the radiation energy (i.e., $\alpha>1$ ), thermal inflation begins at temperature $T_{\text {infl }}$ with Hubble constant $H_{*}$, which are given by

$$
\frac{g_{*} \pi^{2} T_{\mathrm{infl}}^{4}}{30}=\Delta V=\frac{3 H_{*}^{2} M_{\mathrm{pl}}^{2}}{8 \pi} .
$$

During this phase, all particles undergo supercooling, because the scale factor grows exponentially and the temperature falls inversely with the scale factor. Supercooling ends at temperature $T_{\text {end }}$ with a phase transition to the true vacuum at $\langle\eta\rangle=v_{\eta},\langle h\rangle=v_{\mathrm{SM}}$. Supercooling ends when the temperature falls to the nucleation temperature $T_{n}$, or earlier at the QCD phase transition temperature $T_{\mathrm{QCD}}$ if $T_{\mathrm{QCD}}>T_{n}$ :

$$
T_{\text {end }}=\max \left(T_{n}, T_{\mathrm{QCD}}\right), \quad T_{\mathrm{QCD}} \simeq \frac{0.1\langle h\rangle_{\mathrm{QCD}}}{m_{\chi} / \mathrm{TeV}},
$$

where $\langle h\rangle_{\mathrm{QCD}} \simeq 100 \mathrm{MeV}$.

The Coleman-Weinberg mechanism generates a true minimum at $\langle\eta\rangle=v_{\eta}$ when the quartic $\lambda_{S}$ becomes negative at a scale, $v_{\eta} e^{1 / 4}[11]$. Assuming the true vacuum has zero energy, the energy in the false vacuum is $\Delta V \simeq 9 m_{\chi}^{4} /\left(128 \pi^{2}\right)$, which implies that supercooling starts at [11]

$$
T_{\mathrm{infl}} \simeq \frac{m_{\chi}}{8.5} \quad \text { and } \quad H_{*}=\sqrt{\frac{3}{\pi}} \frac{m_{\chi}^{2}}{4 M_{\mathrm{pl}}} .
$$


To compute the GW spectra we take $T_{\star}=T_{\text {infl }}[30]$. To calculate $T_{\mathrm{n}}$, we use the bounce action,

$$
\frac{S_{3}}{T}= \begin{cases}\frac{873.71}{g_{X}^{2.37}\left|\ln \left(0.60 T / v_{\eta}\right)\right|}, & \text { for } g_{X}<1.18 \\ 142 \times \frac{\ln \left(T_{\text {infl }} / v_{\eta}\right)-e^{-4.7979\left(g_{X}-1.1779\right)}}{\ln \left(T / v_{\eta}\right)}, & \text { for } g_{X} \geq 1.18\end{cases}
$$

which exactly reproduces the numerical result in figure 1 of ref. [11]. Nucleation occurs when $S_{3}\left(T_{n}\right) / T_{n} \simeq 4 \ln \left(M_{\mathrm{pl}} / m_{\chi}\right) \simeq 142$.

After inflation ends, the universe is reheated by the transfer of vacuum energy $\Delta V$ from the scalars to the other particles. How quickly this occurs determines the reheating temperature $T_{\mathrm{rh}}$. If the scalars decay rapidly, $T_{\mathrm{rh}} \sim T_{\mathrm{infl}}$, and if they oscillate and transfer energy at a rate $\Gamma$ much slower than the Hubble rate before decaying, $T_{\text {rh }}$ is lower, i.e.,

$$
T_{\mathrm{rh}}=T_{\text {infl }} \min (1, \Gamma / H)^{1 / 2} .
$$

We assume that the energy transfer rate is dominated by Higgs decay, so $\Gamma \simeq$ $\Gamma_{h} \sin ^{2}\left(v_{\mathrm{SM}} / v_{\eta}\right)$, where the Higgs decay width, $\Gamma_{h} \approx 4 \mathrm{MeV}$.

\subsubsection{Dark matter abundance}

Having calculated $T_{\text {infl }}, T_{\text {end }}$ and $T_{\text {rh }}$, we now consider the DM relic abundance in two regimes: $T_{\mathrm{rh}}>T_{\mathrm{dec}}$ and $T_{\mathrm{rh}}<T_{\mathrm{dec}}$, where $T_{\mathrm{dec}}$ is the decoupling temperature in the conventional freeze-out scenario. For $T_{\mathrm{rh}}<T_{\mathrm{dec}}$, the DM abundance is dictated by supercooling and by sub-thermal production via scattering. Although we account for bubble filtering, its effect is negligible. On the other hand, for $T_{\mathrm{rh}}>T_{\mathrm{dec}}$, the supercooled population is washed out, and the sub-thermal population reattains thermal equilibrium and produces the relic abundance as in the standard freeze-out scenario. The $\Omega_{\mathrm{DM}} h^{2}=0.11$ contour in the upper-left corner of figure 5 corresponds to this case.

The DM abundance resulting from inflationary supercooling is

$$
\frac{\left.n_{\mathrm{DM}}\right|_{T=T_{\mathrm{rh}}}}{\left.s\right|_{T=T_{\mathrm{rh}}}}=\frac{45 g_{\mathrm{DM}}}{2 \pi^{4} g_{*}} \frac{T_{\mathrm{rh}}}{T_{\mathrm{infl}}}\left(\frac{T_{\mathrm{end}}}{T_{\mathrm{infl}}}\right)^{3} \times f_{\mathrm{in}},
$$

where $f_{\text {in }} \equiv\left(\left.n_{\chi}^{\text {in }}\right|_{T_{\text {end }}}\right) /\left(\left.n_{\chi}^{\text {eq }}\right|_{T_{\text {end }}}\right)$ quantifies the filtering effect with $T_{\star}=T_{\text {end }}$ in eq. (2.1). However, $f_{\text {in }}=1$ for most of the parameter space of this model because the bubble wall velocity is close to the speed of light and $\gamma_{w} T_{\text {end }} \gg m_{\chi}$. The dilution from supercooling is significant for $T_{\text {infl }} / T_{\text {end }} \gg 1$, and can lead to DM being under-produced; this corresponds to the white region in the lower-right corner of figure 5. The DM density today can be calculated by rescaling from $T_{\text {rh }}$ to the temperature today, $0.235 \mathrm{meV}$, and using eq. (2.2).

We now consider sub-thermal DM production after supercooling. The decoupling temperature of this population is $T_{\text {dec }} \simeq m_{\chi} / \ln \lambda$, where $\lambda \equiv M_{\mathrm{pl}} m_{\chi}\left\langle\sigma_{\mathrm{ann}} v\right\rangle \sqrt{\pi g_{\star} / 45}$, and $\left\langle\sigma_{\text {ann }} v\right\rangle$ is the thermal averaged DM annihilation cross section of the DMDM $\rightarrow \eta \eta$ process [11]. The abundance of the sub-thermal population is obtained by solving the Boltzmann equation. 


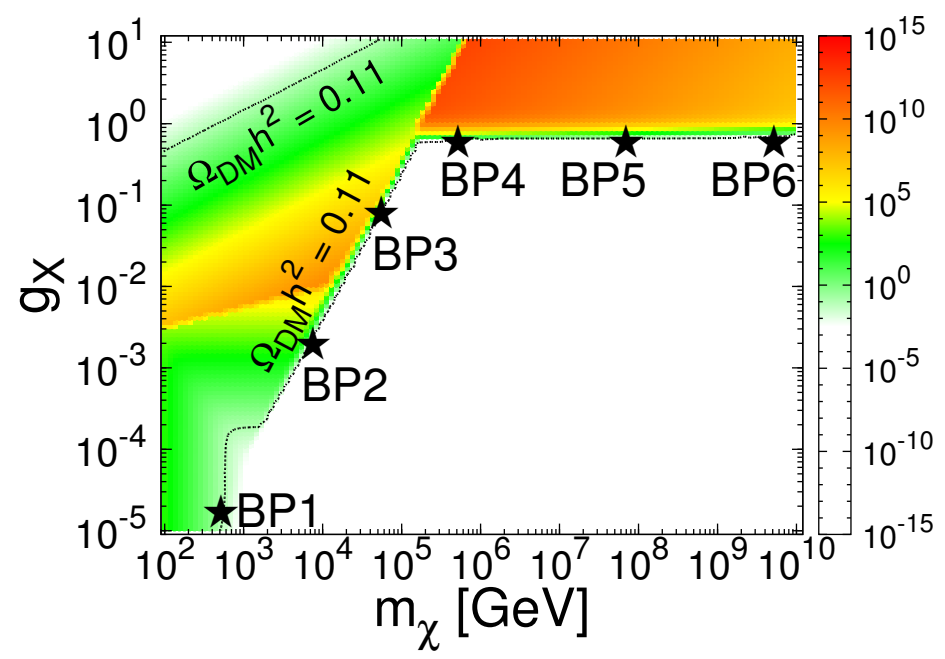

Figure 5. $\Omega_{\mathrm{DM}} h^{2}$ for the $\mathrm{SU}(2)_{X}$ Model with $\langle h\rangle_{\mathrm{QCD}}=100 \mathrm{MeV}$. The black-dashed contours indicate the observed DM relic abundance, $\Omega_{\mathrm{DM}} h^{2}=0.11$. The stars mark the six benchmark points in table 2 .

For $T_{\mathrm{rh}}<T_{\mathrm{dec}}$, both supercooling and sub-thermal production contribute to the DM relic abundance,

$$
\Omega_{\mathrm{DM}} h^{2}=\left.\Omega_{\mathrm{DM}} h^{2}\right|_{\text {supercool }}+\left.\Omega_{\mathrm{DM}} h^{2}\right|_{\text {sub-thermal }} .
$$

For $T_{\mathrm{rh}}>T_{\mathrm{dec}}$, the plasma thermalizes again, and the usual freeze-out mechanism yields the relic abundance,

$$
\Omega_{\mathrm{DM}} h^{2}=\left.\Omega_{\mathrm{DM}} h^{2}\right|_{\text {freeze-out }} \simeq 0.11 \times \frac{\left\langle\sigma_{\mathrm{ann}} v\right\rangle}{2 \times 10^{-26} \mathrm{~cm}^{3} / \mathrm{s}} .
$$

The DM relic abundance is shown in figure 5. We mark six benchmark points along the dashed curves (which indicate $\Omega_{\mathrm{DM}} h^{2} \simeq 0.11$ ), and their values are listed in table 2 . For BP2 and BP3 sub-thermal processes dominate. Dilution by supercooling fixes the DM abundances for BP1, BP4, BP5, and BP6. The end of supercooling occurs at the nucleation temperature for $\mathbf{B P 4}, \mathbf{B P 5}$ and $\mathbf{B P 6}$, and at the QCD phase transition temperature for BP1. For the $\Omega_{\mathrm{DM}} h^{2}=0.11$ contour in the upper-left corner of figure 5 , $T_{\mathrm{rh}}>T_{\mathrm{dec}}$ and the DM abundance is produced by the usual thermal freeze-out. However, we have checked that the pressure difference due to transition radiation across the wall becomes larger than the vacuum pressure driving the bubble expansion [25]. Since this renders our assumption that GWs are predominately produced by bubble collisions invalid, we do not consider this part of the parameter space any further.

\subsubsection{Gravitational wave signals}

To calculate the GW spectra, we need the phase transition strength $\alpha$, inverse phase transition duration $\beta / H_{\star}, v_{w}$, and $T_{\star}$. We evaluate $\alpha$ and $v_{w}$ by following the procedure of section 3 and replacing $T_{\star}$ by $T_{\text {end }}$ in eq. (3.1) to make the equation valid for vacuum transitions [23]. The bounce action is used to find $\beta / H_{\star}=d\left(S_{3} / T\right) /\left.d(\ln T)\right|_{T=T_{\text {end }}}$. We 


\begin{tabular}{|c|cccccc|}
\hline & BP1 & BP2 & BP3 & BP4 & BP5 & BP6 \\
\hline$m_{\chi} / \mathrm{GeV}$ & 540 & $5.4 \times 10^{3}$ & $4.2 \times 10^{4}$ & $3.0 \times 10^{5}$ & $8 \times 10^{7}$ & $6 \times 10^{9}$ \\
$g_{X}$ & $1.7 \times 10^{-5}$ & $1.5 \times 10^{-3}$ & $5.9 \times 10^{-2}$ & 0.72 & 0.77 & 0.82 \\
\hline$\alpha$ & $1.3 \times 10^{14}$ & $1.2 \times 10^{22}$ & $1.8 \times 10^{29}$ & $4.4 \times 10^{16}$ & $2.8 \times 10^{13}$ & $9.4 \times 10^{10}$ \\
$\beta / H_{\star}$ & $3.5 \times 10^{11}$ & $8.1 \times 10^{6}$ & $1.3 \times 10^{3}$ & 10.7 & 12.5 & 14.4 \\
$v_{\eta} / T_{\text {end }}$ & $3.4 \times 10^{9}$ & $3.7 \times 10^{9}$ & $6.0 \times 10^{9}$ & $3.4 \times 10^{5}$ & $5.1 \times 10^{4}$ & $1.2 \times 10^{4}$ \\
$\gamma_{w}$ & $2.9 \times 10^{5}$ & $2.9 \times 10^{7}$ & $1.8 \times 10^{9}$ & $1.2 \times 10^{6}$ & $2.0 \times 10^{5}$ & $4.7 \times 10^{4}$ \\
$T_{\text {end }} / \mathrm{GeV}$ & $1.85 \times 10^{-2}$ & $1.87 \times 10^{-3}$ & $2.4 \times 10^{-4}$ & 2.42 & $4.01 \times 10^{3}$ & $1.27 \times 10^{6}$ \\
$T_{\text {rh }} / \mathrm{GeV}$ & 46.6 & 422 & 2082 & 3566 & 14.5 & 0.201 \\
$T_{\text {infl }} / \mathrm{GeV}$ & 63.6 & 629 & $4.95 \times 10^{3}$ & $3.53 \times 10^{4}$ & $9.29 \times 10^{6}$ & $7.08 \times 10^{8}$ \\
\hline
\end{tabular}

Table 2. Benchmark points for the $\mathrm{SU}(2)_{X}$ Model that give $\Omega_{\mathrm{DM}} h^{2}=0.11$. Note that $T_{\star}=T_{\mathrm{infl}}$.

take $T_{\star}=T_{\text {infl }}$, and rescale the peak frequency by $\left(T_{\mathrm{rh}} / T_{\mathrm{inf}}\right)^{1 / 3}$ and the amplitude by $\left(T_{\mathrm{rh}} / T_{\mathrm{inf}}\right)^{4 / 3}$ to account for a period of matter domination after the phase transition [30]. The values of these parameters are provided in table 2 for the six benchmark points. The extremely large values of $\alpha$ and $\gamma_{w}$ are representative of ultra supercooling, for which the pressure $P$ cannot counter the vacuum energy $\Delta V$, so that bubble expansion keeps accelerating, until the bubbles collide and produce GWs. All our benchmark points satisfy the requirement that $\Delta V$ is much larger than the pressure from transition radiation [30]:

$$
T_{n}^{4} \ll \frac{\beta}{H_{\star}} \frac{m_{\chi}^{5}}{g_{X}^{2} M_{\mathrm{pl}}} .
$$

In figure 6 , we display the GW spectra for a few benchmark points and the sensitivities of the LIGO O2 and O5 observing runs [31], LISA [23, 32], ET [33], BBO [34], and DECIGO [35] are provided for comparison. The BP1, BP2 and BP6 signals are suppressed to an unobservable level because of the large $\beta / H_{\star}$ for $\mathbf{B P 1}$ and $\mathbf{B P 2}$, and small $T_{\mathrm{rh}} / T_{\mathrm{infl}}$ for BP6. BP3 and BP4 produce strong signals at BBO and DECIGO, and BP5 is marginally detectable at BBO. BP4 can also be detected by LISA, and marginally by ET.

\section{Summary}

We studied the sudden freeze-out of DM as an alternative to the continuous thermal freezeout mechanism. A necessary ingredient for sudden freeze-out is that a FOPT generates DM mass. DM mass is generated via the coupling to a scalar particle, whose potential is responsible for a FOPT. When the scalar field acquires a non-zero VEV, DM becomes massive. The DM relic abundance may be determined by bubble filtering or by inflation and reheating. Because a FOPT triggers sudden DM freeze-out, GWs offer a signature for sudden freeze-out not available for thermal freeze-out.

To assess the viability of GWs as a signal of sudden freeze-out, we considered two example models that produce a DM abundance either by bubble-filtering (Scalar Quartic 


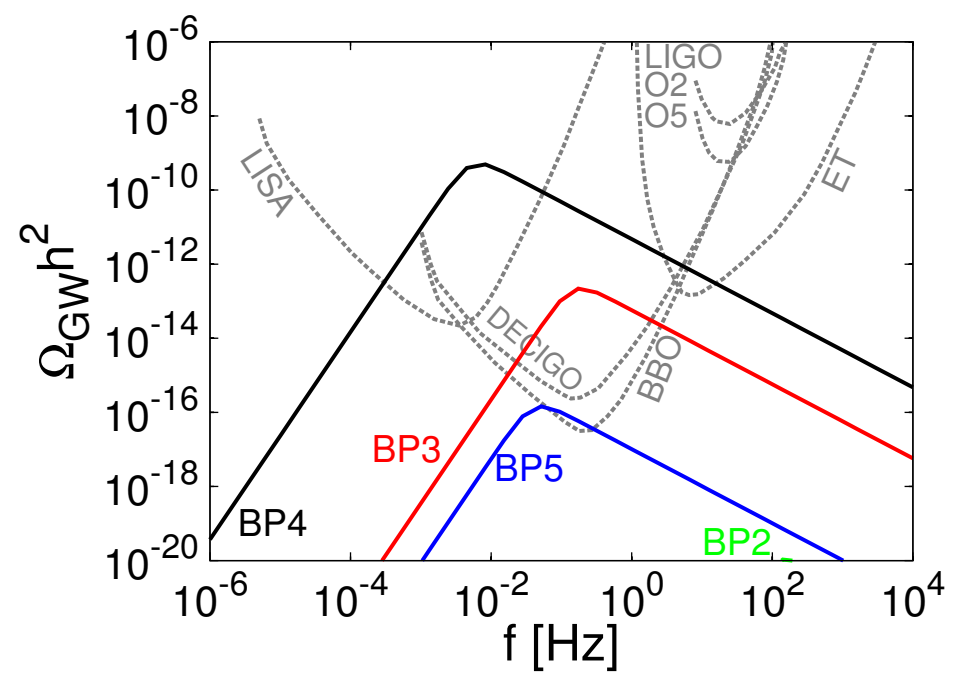

Figure 6. The GW power spectra for benchmark points of the $\mathrm{SU}(2)_{X}$ Model in table 2 and figure 5. The signals of BP1 and BP6 are too small to display.

Model) or by inflation and reheating $\left(\mathrm{SU}(2)_{X}\right.$ Model). We showed that the observed DM relic abundance can be realized in these models with detectable GW signals in future interferometers.

In the Scalar Quartic Model, the perturbativity condition, $g_{\chi} \lesssim \sqrt{4 \pi}$, forces the preferred parameter space to have a large $v_{\eta} / T_{\star} \gtrsim 20$ and small phase transition strength, $\alpha \lesssim 0.2$. To produce the DM relic abundance, the expanding bubbles must filter out most of the thermal DM in the symmetric phase via a large $m_{\chi} / T_{\star}$ and non-relativistic bubble wall velocity. In these parameter regions the $\mathrm{GW}$ spectra have peak frequencies $\mathcal{O}\left(10^{-2}\right) \mathrm{Hz}$, and powers large enough to be probed by LISA, DECIGO, and BBO.

In the $\mathrm{SU}(2)_{X}$ Model, bubble filtering has a negligible effect on the DM number density, and the DM relic abundance is governed either by supercooling during thermal inflation or sub-thermal DM production. The parameter regions that give the DM relic abundance favor $\alpha \gg 1$, which corresponds to ultra supercooling. Therefore, GWs originate from bubble collisions. Observable GW spectra have peak frequencies between about $10^{-3} \mathrm{~Hz}$ to $1 \mathrm{~Hz}$, and enough power to be probed by LISA, BBO, DECIGO and ET. For BP3 and BP4, the GW power is above $\Omega_{\mathrm{GW}} h^{2} \simeq 10^{-13}$.

\section{Acknowledgments}

We thank P. Schwaller and an anonymous referee for useful comments. D.M. is supported in part by the U.S. DOE under Grant No. de-sc0010504.

Open Access. This article is distributed under the terms of the Creative Commons Attribution License (CC-BY 4.0), which permits any use, distribution and reproduction in any medium, provided the original author(s) and source are credited. 


\section{References}

[1] E.W. Kolb, D.J.H. Chung and A. Riotto, WIMPzillas!, AIP Conf. Proc. 484 (1999) 91 [hep-ph/9810361] [INSPIRE].

[2] R. Allahverdi, K. Dutta and A. Maharana, Constraining non-thermal dark matter by CMB, JCAP 10 (2018) 038 [arXiv:1808.02659] [INSPIRE].

[3] J. McDonald, Thermally generated gauge singlet scalars as selfinteracting dark matter, Phys. Rev. Lett. 88 (2002) 091304 [hep-ph/0106249] [INSPIRE].

[4] L.J. Hall, K. Jedamzik, J. March-Russell and S.M. West, Freeze-in production of FIMP dark matter, JHEP 03 (2010) 080 [arXiv:0911.1120] [INSPIRE].

[5] X. Chu, Y. Mambrini, J. Quevillon and B. Zaldivar, Thermal and non-thermal production of dark matter via $Z^{\prime}$-portal(s), JCAP 01 (2014) 034 [arXiv:1306.4677] [InSPIRE].

[6] ATLAS collaboration, Observation of a new particle in the search for the Standard Model Higgs boson with the ATLAS detector at the LHC, Phys. Lett. B 716 (2012) 1 [arXiv: 1207.7214] [INSPIRE].

[7] CMS collaboration, Observation of a new boson at a mass of $125 \mathrm{GeV}$ with the CMS experiment at the LHC, Phys. Lett. B 716 (2012) 30 [arXiv:1207.7235] [INSPIRE].

[8] K. Kajantie, M. Laine, K. Rummukainen and M.E. Shaposhnikov, The electroweak phase transition: a nonperturbative analysis, Nucl. Phys. B 466 (1996) 189 [hep-lat/9510020] [INSPIRE].

[9] K. Kajantie, M. Laine, K. Rummukainen and M.E. Shaposhnikov, Is there a hot electroweak phase transition at $m_{H} \gtrsim m_{W}$ ?, Phys. Rev. Lett. 77 (1996) 2887 [hep-ph/9605288] [INSPIRE].

[10] T. Hambye and A. Strumia, Dynamical generation of the weak and dark matter scale, Phys. Rev. D 88 (2013) 055022 [arXiv: 1306.2329] [INSPIRE].

[11] T. Hambye, A. Strumia and D. Teresi, Super-cool dark matter, JHEP 08 (2018) 188 [arXiv: 1805.01473] [INSPIRE].

[12] L. Heurtier and H. Partouche, Spontaneous freeze out of dark matter from an early thermal phase transition, Phys. Rev. D 101 (2020) 043527 [arXiv:1912.02828] [INSPIRE].

[13] M.J. Baker, J. Kopp and A.J. Long, Filtered dark matter at a first order phase transition, Phys. Rev. Lett. 125 (2020) 151102 [arXiv: 1912.02830] [InSPIRE].

[14] D. Chway, T.H. Jung and C.S. Shin, Dark matter filtering-out effect during a first-order phase transition, Phys. Rev. D 101 (2020) 095019 [arXiv:1912.04238] [INSPIRE].

[15] J. Kehayias and S. Profumo, Semi-analytic calculation of the gravitational wave signal from the electroweak phase transition for general quartic scalar effective potentials, JCAP 03 (2010) 003 [arXiv:0911.0687] [INSPIRE].

[16] X. Wang, F.P. Huang and X. Zhang, Phase transition dynamics and gravitational wave spectra of strong first-order phase transition in supercooled universe, JCAP 05 (2020) 045 [arXiv:2003.08892] [INSPIRE].

[17] M. Dine, R.G. Leigh, P.Y. Huet, A.D. Linde and D.A. Linde, Towards the theory of the electroweak phase transition, Phys. Rev. D 46 (1992) 550 [hep-ph/9203203] [INSPIRE]. 
[18] F.C. Adams, General solutions for tunneling of scalar fields with quartic potentials, Phys. Rev. D 48 (1993) 2800 [hep-ph/9302321] [INSPIRE].

[19] J.R. Espinosa, T. Konstandin, J.M. No and G. Servant, Energy budget of cosmological first-order phase transitions, JCAP 06 (2010) 028 [arXiv: 1004.4187] [INSPIRE].

[20] D. Bödeker and G.D. Moore, Can electroweak bubble walls run away?, JCAP 05 (2009) 009 [arXiv: 0903.4099] [INSPIRE].

[21] P.J. Steinhardt, Relativistic detonation waves and bubble growth in false vacuum decay, Phys. Rev. D 25 (1982) 2074 [INSPIRE].

[22] M. Laine, Bubble growth as a detonation, Phys. Rev. D 49 (1994) 3847 [hep-ph/9309242] [INSPIRE].

[23] C. Caprini et al., Science with the space-based interferometer eLISA. II: gravitational waves from cosmological phase transitions, JCAP 04 (2016) 001 [arXiv: 1512.06239] [INSPIRE].

[24] S.J. Huber and T. Konstandin, Gravitational wave production by collisions: more bubbles, JCAP 09 (2008) 022 [arXiv: 0806.1828] [INSPIRE].

[25] D. Bödeker and G.D. Moore, Electroweak bubble wall speed limit, JCAP 05 (2017) 025 [arXiv: 1703.08215] [INSPIRE].

[26] M. Heikinheimo, A. Racioppi, M. Raidal, C. Spethmann and K. Tuominen, Physical naturalness and dynamical breaking of classical scale invariance, Mod. Phys. Lett. A 29 (2014) 1450077 [arXiv: 1304.7006] [INSPIRE].

[27] M. Heikinheimo, A. Racioppi, M. Raidal, C. Spethmann and K. Tuominen, Dark supersymmetry, Nucl. Phys. B 876 (2013) 201 [arXiv:1305.4182] [INSPIRE].

[28] T. Hambye, Hidden vector dark matter, JHEP 01 (2009) 028 [arXiv:0811.0172] [INSPIRE].

[29] M. Cirelli, N. Fornengo and A. Strumia, Minimal dark matter, Nucl. Phys. B 753 (2006) 178 [hep-ph/0512090] [INSPIRE].

[30] I. Baldes and C. Garcia-Cely, Strong gravitational radiation from a simple dark matter model, JHEP 05 (2019) 190 [arXiv: 1809.01198] [INSPIRE].

[31] KAGRA, LigO Scientific and Virgo collaborations, Prospects for observing and localizing gravitational-wave transients with advanced LIGO, advanced Virgo and KAGRA, Living Rev. Rel. 21 (2018) 3 [arXiv:1304.0670] [INSPIRE].

[32] P. Auclair et al., Probing the gravitational wave background from cosmic strings with LISA, JCAP 04 (2020) 034 [arXiv: 1909.00819] [INSPIRE].

[33] S. Hild et al., Sensitivity studies for third-generation gravitational wave observatories, Class. Quant. Grav. 28 (2011) 094013 [arXiv: 1012.0908] [INSPIRE].

[34] K. Yagi and N. Seto, Detector configuration of DECIGO/BBO and identification of cosmological neutron-star binaries, Phys. Rev. D 83 (2011) 044011 [Erratum ibid. 95 (2017) 109901] [arXiv: 1101.3940] [INSPIRE].

[35] DECIGO WORKING GRoup collaboration, Primordial gravitational wave and DECIGO, PoS (KMI2019) 019 (2019) [INSPIRE]. 